\title{
Analytical Computation of Impedance Integrals with Power-Law Green's Functions
}

\author{
I. Bogaert*
}

\begin{abstract}
In this contribution, a method is presented for reducing the number of subsequent integrations that occur in impedance integrals with Green's functions of the form $R^{\nu}$, with $R$ the distance between source and observation point. The method allows the number of integrations to be reduced to 1 in the two dimensional case and 2 in the three dimensional case, irrespective of the number of subsequent integrations that were originally present. These last integrations can be done analytically using well-known results if $\nu \in \mathbb{Z}$, resulting in a computation that is free of numerical integrations. The dynamic Green's function can be treated in a semianalytical way, by expanding it into a Taylor series in the wavenumber. The method can be applied if both the basis and test functions are polynomial functions with polygonal support and if certain non-parallelity conditions are satisfied.
\end{abstract}

\section{INTRODUCTION}

An important class of impedance integrals arising from the discretization of integral equations are of the form

$$
Z=\int_{V_{t}} \int_{V_{b}} t(\boldsymbol{r})\left\|\boldsymbol{r}-\boldsymbol{r}^{\prime}\right\|^{\nu} b\left(\boldsymbol{r}^{\prime}\right) \mathrm{d} \boldsymbol{r}^{\prime} \mathrm{d} \boldsymbol{r},
$$

with basis function $b\left(\boldsymbol{r}^{\prime}\right)$, test function $t(\boldsymbol{r})$ and Green's function $\left\|\boldsymbol{r}-\boldsymbol{r}^{\prime}\right\|^{\nu}$. The integration domains $V_{b}$ and $V_{t}$ denote the support of the basis and test function respectively. Even today, the computation of impedance integrals such as (1) is a challenging task, because of the presence of singularities in the integrand. This singularity makes it difficult to get a good accuracy using standard numerical integration rules. At the same time, many impedance integrals have to be computed for even the simplest scattering problem, which means that the computation procedure should be fast. This requirement precludes the use of adaptive or other general-purpose techniques for handling the singular integrands. Therefore, a large body of research has been devoted to the development of methods that are specifically tailored for the computation of impedance integrals, usually whilst striking a balance between accuracy and speed.

To illustrate this, we will briefly review some of the methods that have been developed in the past,

\footnotetext{
* Department of Information Technology, Ghent University, Sint-Pietersnieuwstraat 41, 9000 Ghent, Belgium, email:Ignace.Bogaert@intec.UGent . be
}

without trying to give an exhaustive list. One of the earliest, and most widely used, methods is to use analytical techniques to compute the inner integral [1]

$$
F(\boldsymbol{r})=\int_{V_{b}}\left\|\boldsymbol{r}-\boldsymbol{r}^{\prime}\right\|^{\nu} b\left(\boldsymbol{r}^{\prime}\right) \mathrm{d} \boldsymbol{r}^{\prime},
$$

and evaluate the remaining integral by means of numerical quadrature rules. This technique can also be used for the dynamic Green's function, using a technique called singularity extraction [2-4]. A disadvantage of this technique is that $F(\boldsymbol{r})$ can still have non-smooth features, such as the presence of singular derivatives for $\nu=-1$ when $V_{b}$ and $V_{t}$ share a part of their support. A solution to this problem has been proposed in [5], by using a double-exponential-based quadrature rule for the outer integration. This scheme results in a wellcontrolled error, as opposed to when a direct product Gauss-Legendre quadrature rule would be used. Alternatively, a number of so-called singularity cancelation methods have been proposed [6-9] for the evaluation of (2), that avoid the need for analytical results and can be extended to basis functions with curvilinear support.

It is clear that the methods mentioned above involve a trade-off between accuracy and speed. Such a trade-off could be avoided if analytical expressions could be found for impedance integrals of the type (1). Unfortunately, to the best knowledge of the authors, no such expressions have been proposed yet in the literature. Nevertheless, significant advances have been made. For example, in 1997, a closed-form expression was found for the complete self-patch integral with $\nu=-1$ [10]. This was extended to various other integer $\nu$ in [11]. Recently, the case for general triangles was solved for basis functions proportional to the position vector [12] and constant test functions (or vice versa).

In this contribution, a general methodology is proposed for the reduction of the number of successive integrations in impedance integrals. It is based on a re-scaling of the coordinate system and can reduce the number of integrations to 1 in the two dimensional case and 2 in the three dimensional case, irrespective of the number of subsequent integrations that were originally present. The remaining integrations can be performed using well-known 
results $[1,3]$ if $\nu \in \mathbb{Z}$, such that the method can be used to obtain closed form expressions for integrals of the type (1). It should be noted that the method suffers from a numerical instability if the center point of the re-scaling is located very far away from the integration domains $V_{b}$ and $V_{t}$. However, it is expected that this problem can be solved by taking a suitable limit, which is the aim of future work.

\section{RE-SCALING FOR THE COMPUTA- TION OF IMPEDANCE INTEGRALS}

In this contribution, the proposed technique will be used on a simple example where the basis function is a constant (pulse) function with triangular support $V_{b}=\left\{\boldsymbol{r}=u_{b} \boldsymbol{b}_{1}+v_{b} \boldsymbol{b}_{2}+\left(1-u_{b}-v_{b}\right) \boldsymbol{b}_{3}\right.$ : $\left.0 \leq u_{b} \leq 1,0 \leq v_{b} \leq 1-u_{b}\right\}$ and the test function is a constant function on a line $V_{t}=\left\{\boldsymbol{r}=u_{t} \boldsymbol{t}_{1}+\right.$ $\left.\left(1-u_{t}\right) \boldsymbol{t}_{2}: 0 \leq u_{t} \leq 1\right\}$. Such an impedance integral might for example occur when analyzing a scattering problem with both surfaces and wires. This example was chosen because it is simpler than the triangle-triangle case, and much simpler than the tetrahedron-tetrahedron case. Therefore, it allows us to more fully focus on the ideas relevant to the proposed method and avoid an overburdened notation.

Figure 1 shows a typical configuration for a triangle and line. The reduction of the number of subsequent integrations hinges on finding a point $\boldsymbol{p}$ that is located both along the extension of the line and in the plane of the triangle. Clearly, this is possible whenever the line is not parallel to the plane of the triangle, or when the line and triangle are coplanar. Of course, it can also happen that the line is parallel to the triangle, but not coplanar. In that case, the sought point $\boldsymbol{p}$ is located at infinity. However, for now, it will be assumed that a finite $\boldsymbol{p}$ can be found, and we will return to the infinite $\boldsymbol{p}$ case later on.

For the case at hand (triangle-line interaction, constant basis and test functions), integral (1) can be simplified to

$$
Z=\int_{0}^{1} \int_{0}^{1} \int_{0}^{1-u_{b}}\left\|\boldsymbol{r}\left(u_{t}\right)-\boldsymbol{r}^{\prime}\left(u_{b}, v_{b}\right)\right\|^{\nu} \mathrm{d} v_{b} \mathrm{~d} u_{b} \mathrm{~d} u_{t} .
$$

Also, because of the definition of the point $\boldsymbol{p}$, there exist values $u_{b 0}, v_{b 0}$ and $u_{t 0}$ such that

$$
\begin{aligned}
\boldsymbol{p} & =u_{b 0} \boldsymbol{b}_{1}+v_{b 0} \boldsymbol{b}_{2}+\left(1-u_{b 0}-v_{b 0}\right) \boldsymbol{b}_{3} \\
& =u_{t 0} \boldsymbol{t}_{1}+\left(1-u_{t 0}\right) \boldsymbol{t}_{2} .
\end{aligned}
$$

This information can be used to do the following scaling

$$
u_{b} \rightarrow u_{b}+\Delta\left(u_{b}-u_{b 0}\right)
$$

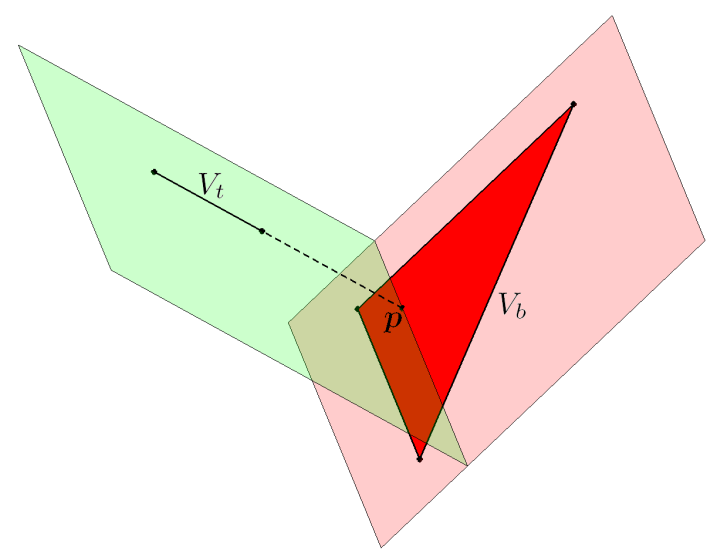

Figure 1: An example configuration of a triangle and a line. The point $\boldsymbol{p}$ is located both along the extension of the line and in the plane of the triangle.

$$
\begin{aligned}
& v_{b} \rightarrow v_{b}+\Delta\left(v_{b}-v_{b 0}\right), \\
& u_{t} \rightarrow u_{t}+\Delta\left(u_{t}-u_{t 0}\right) .
\end{aligned}
$$

When this is substituted in (3), the following is obtained after some simplifications

$$
\begin{aligned}
Z=\gamma^{\nu+3} \int_{\frac{\Delta u_{t 0}}{\gamma}}^{\frac{1+\Delta u_{t 0}}{\gamma}} \int_{\frac{\Delta u_{b 0}}{\gamma}}^{\frac{1+\Delta u_{b 0}}{\gamma}} \int_{\frac{\Delta v_{b 0}}{\gamma}}^{\frac{1+\Delta\left(u_{b 0}+v_{b 0}\right)}{\gamma}-u_{b}} \\
\\
\left\|\boldsymbol{r}\left(u_{t}\right)-\boldsymbol{r}^{\prime}\left(u_{b}, v_{b}\right)\right\|^{\nu} \mathrm{d} v_{b} \mathrm{~d} u_{b} \mathrm{~d} u_{t}
\end{aligned}
$$

with

$$
\gamma=1+\Delta
$$

Expression (6) holds for general $\Delta$. Therefore, it is possible to evaluate the derivative of (6) with respect to $\Delta$ in zero. Because the left hand side does not depend on $\Delta$, the following is found:

$$
\begin{aligned}
& (\nu+3) Z= \\
& u_{t 0} \int_{0}^{1} \int_{0}^{1-u_{b}}\left\|\boldsymbol{r}(0)-\boldsymbol{r}^{\prime}\left(u_{b}, v_{b}\right)\right\|^{\nu} \mathrm{d} v_{b} \mathrm{~d} u_{b} \\
& +v_{t 0} \int_{0}^{1} \int_{0}^{1-u_{b}}\left\|\boldsymbol{r}(1)-\boldsymbol{r}^{\prime}\left(u_{b}, v_{b}\right)\right\|^{\nu} \mathrm{d} v_{b} \mathrm{~d} u_{b} \\
& +u_{b 0} \int_{0}^{1} \int_{0}^{1}\left\|\boldsymbol{r}\left(u_{t}\right)-\boldsymbol{r}^{\prime}\left(0, v_{b}\right)\right\|^{\nu} \mathrm{d} v_{b} \mathrm{~d} u_{t} \\
& +v_{b 0} \int_{0}^{1} \int_{0}^{1}\left\|\boldsymbol{r}\left(u_{t}\right)-\boldsymbol{r}^{\prime}\left(u_{b}, 0\right)\right\|^{\nu} \mathrm{d} u_{b} \mathrm{~d} u_{t} \\
& +w_{b 0} \int_{0}^{1} \int_{0}^{1}\left\|\boldsymbol{r}\left(u_{t}\right)-\boldsymbol{r}^{\prime}\left(u_{b}, 1-u_{b}\right)\right\|^{\nu} \mathrm{d} u_{b} \mathrm{~d} u_{t},
\end{aligned}
$$

with

$$
\begin{aligned}
v_{t 0} & =1-u_{t 0}, \\
w_{b 0} & =1-u_{b 0}-v_{b 0} .
\end{aligned}
$$


It is clear that (8) expresses the triple integral (3) as a sum of five double integrals, for general $\nu \in \mathbb{C}$. If $\nu \in \mathbb{Z}$, these integrals can be computed analytically using known results. More importantly, the technique of introducing the scaling (5) can be used with the same success in integrals with more subsequent integrations. The number of integrations can then be reduced until no point $\boldsymbol{p}$ that satisfies the analogue of equation (4) can be found any more. In general, this happens when the number of integrations has dropped to two. A very nice property of the scheme presented here is the fact that the Green's function that occurs in (3) is preserved in the two-dimensional integrals of (8). This is also a general characteristic, not limited to the special case considered here.

Finally, it is worthwhile to point out that (8) works for almost completely general triangles and lines. It is only for an easily spotted special case that the formula becomes unstable: if $\boldsymbol{p}$ is located very far (or infinitely far) away from the interacting triangle and line, the factors in front of the double integrals in (8) become very large. This causes a numerical cancelation and therefore a loss of precision. This problem happens when the line is (almost) parallel to the triangle, but not coplanar. In this case, the solution is to tilt the line or triangle by a small amount $\epsilon$. Then, a finite $\epsilon$-dependent point $\boldsymbol{p}_{\epsilon}$ can again be found, for which the analytical formulas can be constructed. Finally, the limit for $\epsilon \rightarrow 0$ must be taken to recover formulas for this special case. This is a topic of future research. Note that this pathological case never occurs for impedance integrals between polygons that touch each other (e.g. self patch or neighbor patch). Therefore, the most challenging, singular, integrals can always be treated using the method proposed here.

\section{NUMERICAL RESULTS}

The formula (8) was tested on a triangle and line with vertices

$$
\begin{aligned}
\boldsymbol{b}_{1} & =[0,0,0], \\
\boldsymbol{b}_{2} & =[1,0,0], \\
\boldsymbol{b}_{3} & =[0,1,1], \\
\boldsymbol{t}_{1} & =[0.2,-0.5,0.7], \\
\boldsymbol{t}_{2} & =[0.2,-1,1.3] .
\end{aligned}
$$

First, formula (8) was leveraged, and its five terms were computed numerically for various values of $\nu \in$ $[-2,4]$. This was compared with a direct numerical evaluation of (3). Figure 2 shows the absolute value $|Z|$ of both results and their difference. Clearly, the accuracy is as good as can be expected in a numerical comparison: the relative error is below $4 \times 10^{-13}$ for all sampled $\nu$.

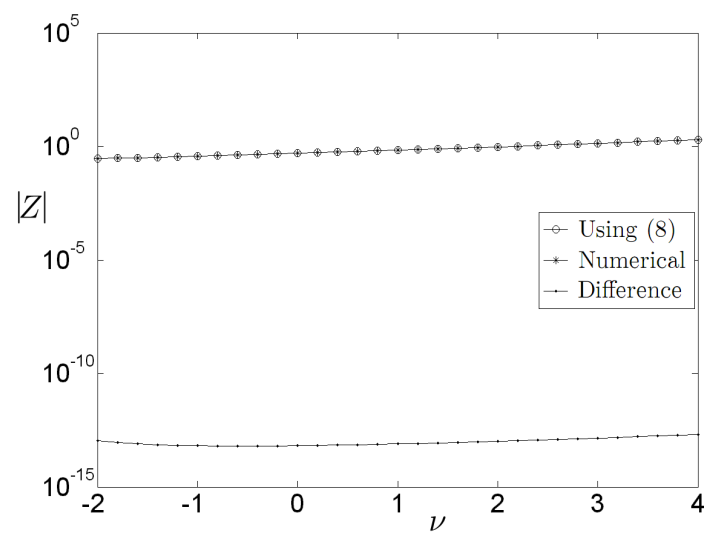

Figure 2: A comparison between the numerical values for the integral $Z$ obtained using formula (8) and a fully numerical quadrature. The absolute value of the difference is also given.

\section{Conclusion}

A general methodology was presented for reducing the number of successive integrations in impedance integrals. It was illustrated by reducing triple integral to a sum of five double integrals. This scheme works for general polynomial basis and test functions on polygonal support, with the exception of cases in which the basis and test polygons are parallel.

\section{Acknowledgments}

This work was supported by a postdoctoral grant from the Fund for Scientific Research Flanders (FWO-Vlaanderen).

\section{References}

[1] D. R. Wilton, S. M. Rao, D. H. S. A. W. Glisson, O. M. Al-Bundak, and C. M. Butler, "Potential integrals for uniform and linear source distributions on polygonal and polyhedral domains," IEEE Trans. Antennas Propag., vol. AP-32, no. 3, Mar. 1984.

[2] C. Butler, "Evaluation of potential integral at singularity of exact kernel in thin-wire calculations," Antennas and Propagation, IEEE Transactions on, vol. 23, no. 2, pp. $293-295$, mar 1975. 
[3] S. Jarvenpaa, M. Taskinen, and P. Yla-Oijala, "Singularity subtraction technique for highorder polynomial vector basis functions on planar triangles," Antennas and Propagation, IEEE Transactions on, vol. 54, no. 1, pp. $42-$ 49, jan. 2006.

[4] I. Hanninen, M. Taskinen, and J. Sarvas, "Singularity subtraction integral formulae for surface integral equations with rwg, rooftop and hybrid basis functions," Progress In Electromagnetics Research, vol. 63, pp. 243-278, 2006.

[5] A. Polimeridis, I. Koufogiannis, M. Mattes, and J. Mosig, "Considerations on double exponential-based cubatures for the computation of weakly singular galerkin inner products," Antennas and Propagation, IEEE Transactions on, vol. 60, no. 5, pp. $2579-2582$, may 2012.

[6] M. A. Khayat and D. R. Wilton, "Numerical Evaluation of Singular and Near-Singular Potential Integrals," IEEE Transactions on Antennas and Propagation, vol. 53, pp. 31803190, Oct. 2005.

[7] M. Khayat, D. Wilton, and P. Fink, "An improved transformation and optimized sampling scheme for the numerical evaluation of singular and near-singular potentials," Antennas and Wireless Propagation Letters, IEEE, vol. 7, pp. $377-380,2008$.

[8] D. Taylor, "Accurate and efficient numerical integration of weakly singular integrals in galerkin efie solutions," Antennas and Propagation, IEEE Transactions on, vol. 51, no. 7, pp. 1630 - 1637, july 2003.

[9] Ismatullah and T. Eibert, "Adaptive singularity cancellation for efficient treatment of nearsingular and near-hypersingular integrals in surface integral equation formulations," $A n$ tennas and Propagation, IEEE Transactions on, vol. 56, no. 1, pp. $274-278$, jan. 2008.

[10] P. Arcioni, M. Bressan, and L. Perregrini, "On the evaluation of the double surface integrals arising in the application of the boundary integral method to 3-d problems," Microwave Theory and Techniques, IEEE Transactions on, vol. 45, no. 3, pp. $436-439$, mar 1997.

[11] I. Bogaert and D. De Zutter, "High precision evaluation of the selfpatch integral for linear basis functions on flat triangles," IEEE Transactions on Antennas and Propagation, vol. 58, no. 5, pp. 1813-1816, May 2010.

[12] M. Taskinen, "On the fully analytical integration of singular double integrals arising from the integral equation methods," in Computational Electromagnetics International Workshop (CEM), 2011, aug. 2011, pp. $13-18$. 